\title{
Three-Dimensional Modeling of the Human Eye Based on Magnetic Resonance Imaging
}

\author{
Krish D. Singh, ${ }^{1}$ Nicola S. Logan, ${ }^{2}$ and Bernard Gilmartin ${ }^{2}$
}

Purpose. A methodology for noninvasively characterizing the three-dimensional (3-D) shape of the complete human eye is not currently available for research into ocular diseases that have a structural substrate, such as myopia. A novel application of a magnetic resonance imaging (MRI) acquisition and analysis technique is presented that, for the first time, allows the 3-D shape of the eye to be investigated fully.

Methods. The technique involves the acquisition of a $T_{2^{-}}$ weighted MRI, which is optimized to reveal the fluid-filled chambers of the eye. Automatic segmentation and meshing algorithms generate a 3-D surface model, which can be shaded with morphologic parameters such as distance from the posterior corneal pole and deviation from sphericity. Full details of the method are illustrated with data from 14 eyes of seven individuals. The spatial accuracy of the calculated models is demonstrated by comparing the MRI-derived axial lengths with values measured in the same eyes using interferometry.

Results. The color-coded eye models showed substantial variation in the absolute size of the 14 eyes. Variations in the sphericity of the eyes were also evident, with some appearing approximately spherical whereas others were clearly oblate and one was slightly prolate. Nasal-temporal asymmetries were noted in some subjects.

Conclusions. The MRI acquisition and analysis technique allows a novel way of examining 3-D ocular shape. The ability to stratify and analyze eye shape, ocular volume, and sphericity will further extend the understanding of which specific biometric parameters predispose emmetropic children subsequently to develop myopia. (Invest Ophthalmol Vis Sci. 2006; 47:2272-2279) DOI:10.1167/iovs.05-0856

$\mathrm{M}$ yopia is the most prevalent of a range of pathologic and developmental conditions that modify the morphology of the eye. ${ }^{1}$ The percentage of young adolescents diagnosed with myopia has increased substantially during the past five decades in both Western (25\%) and East Asian (75\%) industrialized

From the ${ }^{1}$ Cardiff University Brain and Repair Imaging Centre (CUBRIC) and School of Psychology, Cardiff University, Cardiff, Wales, United Kingdom; and the ${ }^{2}$ School of Life and Health Sciences, Aston University, Birmingham, United Kingdom.

Supported by the Lord Dowding Fund for Humane Research and Advantage, West Midlands, which provides funds for the Aston University MRI system, by which all data were collected. The work presented here is the subject of an International Patent Application, number PCT/GB2005/004577, on behalf of all three authors.

Submitted for publication July 4, 2005; revised November 8 and December 22, 2005; accepted March 30, 2006.

Disclosure: K.D. Singh, P; N.S. Logan, P; B. Gilmartin, P

The publication costs of this article were defrayed in part by page charge payment. This article must therefore be marked "advertisement" in accordance with 18 U.S.C. $\$ 1734$ solely to indicate this fact.

Corresponding author: Krish D. Singh, CUBRIC and School of Psychology, Cardiff University, Tower Building, Park Place, Cardiff CF10 3AT, UK; singhkd@cardiff.ac.uk. societies. ${ }^{2}$ Since 2000 , surveys in collaboration with the World Health Organization have used standardized epidemiologic protocols to compare prevalence levels in children aged 5 to 15 years for urban and rural areas across the world. ${ }^{3-7} \mathrm{~A}$ comprehensive analysis of these and other published surveys has recently suggested that the increase in the prevalence of juvenile-onset myopia is more a consequence of increased exposure to educational activity and urbanization than to an inherent genetic or ethnic predisposition. ${ }^{2}$

Although the debate concerning the respective roles of heredity and environment in the etiology of myopia has continued over the past decade, ${ }^{8}$ it is clear that myopia occurs when the refractive components of the anterior segment of the eye fail to compensate for excessive growth of the posterior vitreous chamber, so that the eye outgrows its refractive capability. ${ }^{9,10}$ There is a compelling need to understand why the homeostatic mechanisms regulating normal ocular growth between 6 and 14 years of age should fail in such a high proportion of children, ${ }^{11,12}$ as their myopia will be a lifelong condition that carries a considerable economic burden and, for levels greater than $6 \mathrm{D}$, significantly increased risk of ocular disease. ${ }^{13}$

The seminal observation in 1977 of Wiesel and Raviola ${ }^{14}$ that lid fusion could cause substantial eye enlargement in monkey has subsequently generated fundamental insights into the nature of structural change after induced myopia. ${ }^{15-20}$ However, Stone and Flitcroft ${ }^{21}$ have recently noted that the most commonly studied index of structural change-namely, refractive error and its correlated change with axial length, has significant limitations. They propose that studying the complete three-dimensional (3-D) conformation of the eye may help resolve many of the ambiguities of contemporary refractive research. In particular, Stone and Flitcroft posed the question: Is eye shape a regulated variable, intrinsically coupled to the mechanisms modulating refractive development, and could stratifying eyes by shape provide a basis for an improved mechanistic understanding of the pathophysiology of ametropia in human populations? ${ }^{21}$

Despite this demonstrated need, a methodology for completely and noninvasively characterizing the 3-D shape of the whole human eye is not currently available. Magnetic resonance imaging (MRI) offers the possibility of such a technique, but to date, there have been relatively few studies in which MRI was used to study ocular shape in three dimensions. In a mixed population $(n=15)$ of myopes, emmetropes, and hyperopes, Chen et al. ${ }^{22}$ manually delineated the boundaries of the posterior chamber on a single 3-mm-thick axial slice through the center of the eye, enabling them to quantify shape parameters such as radius and deviations from sphericity on this 2-D plane. Similarly, in a small study, Cheng et al. ${ }^{23}$ acquired MRI images as slices in three dimensions. Using three orthogonal 3-mm-thick slices, they characterized the shape of hyperopic and myopic eyes by measuring the three principal dimensions of the eye (anterior-posterior, equatorial and superior-inferior). Chau et al. ${ }^{24}$ used MRI to demonstrate that there was no relationship between ocular and orbit volumes in 
TABLE 1. A Comparison of Ocular Volumes and Axial Lengths Determined by MRI and Axial Lengths Measured by PCI

\begin{tabular}{|c|c|c|c|c|c|c|}
\hline Participant & Eye & $\begin{array}{c}\text { Mean Sphere Refractive } \\
\text { Error/Diopter }\end{array}$ & $\begin{array}{l}\text { Volume } \\
\left(\mathrm{mm}^{3}\right)\end{array}$ & $\begin{array}{l}\text { MRI Axial Length } \\
\text { (mm) }\end{array}$ & $\begin{array}{l}\text { PCI Axial Length } \\
\text { (mm) }\end{array}$ & $\begin{array}{l}\text { MRI-PCI } \\
(\mathbf{m m})\end{array}$ \\
\hline \multirow[t]{2}{*}{ S1: male; 27 y; white } & Right & +3.25 & 6383.50 & 23.52 & 23.02 & 0.50 \\
\hline & Left & +3.50 & 6307.00 & 23.41 & 22.78 & 0.63 \\
\hline \multirow[t]{2}{*}{ S2: female; $33 \mathrm{y}$; white } & Right & -3.00 & 6497.24 & 24.45 & 23.25 & 1.20 \\
\hline & Left & -4.00 & 6648.03 & 24.40 & 23.65 & 0.75 \\
\hline \multirow[t]{2}{*}{ S3: male; 36 y; white } & Right & +4.00 & 4762.25 & 21.90 & 21.72 & 0.18 \\
\hline & Left & +0.50 & 5334.50 & 22.97 & 22.93 & 0.04 \\
\hline \multirow[t]{2}{*}{ S4: female; 24 y; white } & Right & -9.00 & 7870.75 & 26.15 & 25.88 & 0.27 \\
\hline & Left & -7.00 & 7589.75 & 24.94 & 25.25 & -0.31 \\
\hline \multirow[t]{2}{*}{ S5: female; 24 y; white } & Right & -0.12 & 7255.00 & 23.72 & 23.86 & -0.14 \\
\hline & Left & -0.37 & 7383.50 & 24.36 & 23.86 & 0.50 \\
\hline \multirow{2}{*}{ S6: male; $38 \mathrm{y}$; white/Asian } & Right & -7.00 & 10541.00 & 28.57 & 27.93 & 0.64 \\
\hline & Left & -6.75 & 10136.00 & 28.45 & 27.51 & 0.94 \\
\hline \multirow[t]{2}{*}{ S7: female; 38 y; white, Marfan } & Right & -16.25 & 9798.10 & 27.17 & 26.65 & 0.52 \\
\hline & Left & -15.50 & 9877.10 & 26.58 & 26.54 & 0.04 \\
\hline Mean (SD) & & & & $25.04(2.04)$ & 24.63 (1.97) & 0.41 \\
\hline
\end{tabular}

a population varying from hyperopia to high myopia. Although the volume of the eye was quantified from 3-D MRI data, no attempt was made to characterize the shape of the eye itself. In a recent larger, MRI study $(n=88)$ of myopia, Atchison et al. ${ }^{25}$ measured the three linear dimensions of each eye by careful placement of single, 3-mm-thick, slices in each of the axial and sagittal planes. In a more recent study of the same data, ${ }^{26} 3-\mathrm{D}$ ellipsoids were fitted to the retinal surface identified on twodimensional (2-D) axial and sagittal image sections. Because of the signal-to-noise limitations, none of these studies has reconstructed the complete 3-D structure of the eye from MRI data that have a high resolution in all three dimensions. Rather, they have inferred the 3-D shape of the eye from a very limited number of 2-D sections through the eye.

With the advent of the current generation of high-field MRI systems and newer designs of radio-frequency coils, the acquisition of volumetric ocular MRI data that have high intrinsic resolution in all three dimensions, becomes more feasible. This then allows the construction of a fully 3-D parametric representation of the whole of the surface of the eye. Such a surface model offers new opportunities for studying parameters such as ocular stretching in myopia, both locally and globally across the eye, in individuals and patients. In the current report, a new optimized scan sequence and image analysis technique is presented that allows the generation of such a 3-D model for the eye of any individual. The procedure involves the acquisition of a $\mathrm{T}_{2}$-weighted MRI, which has been optimized to reveal the fluid-filled chambers of the eye. This image can be automatically segmented and meshed to provide a complete 3-D surface model, which can be color-coded with important structural parameters such as accurate and precise measurements of internal linear distances (unaffected by variations in refractive indices and off-axis aberrations) and deviations from sphericity. The visualizations of the color-coded models can be freely rotated in space to inspect any aspect of their configuration.

\section{MethodS}

\section{MRI Acquisition}

Seven participants, chosen to have a variety of refractive errors from myopia to hyperopia, were scanned with a whole-body MRI scanner
FIGURE 1. A schematic describing the analysis steps of the methodology: (a) initial analysis of the $\mathrm{T}_{2}$ weighted images; (b) labeling of voxels in each eye by using a 3-D floodfilling algorithm; (c) construction of a model using a mesh of 32,768 triangular polygons; (d) wrapping of the polygonal model to approximate the shape of the eye; (e) smoothing of the mesh by using local averaging of the vertex positions; and (f) the visualization of the parameterized 3-D models.

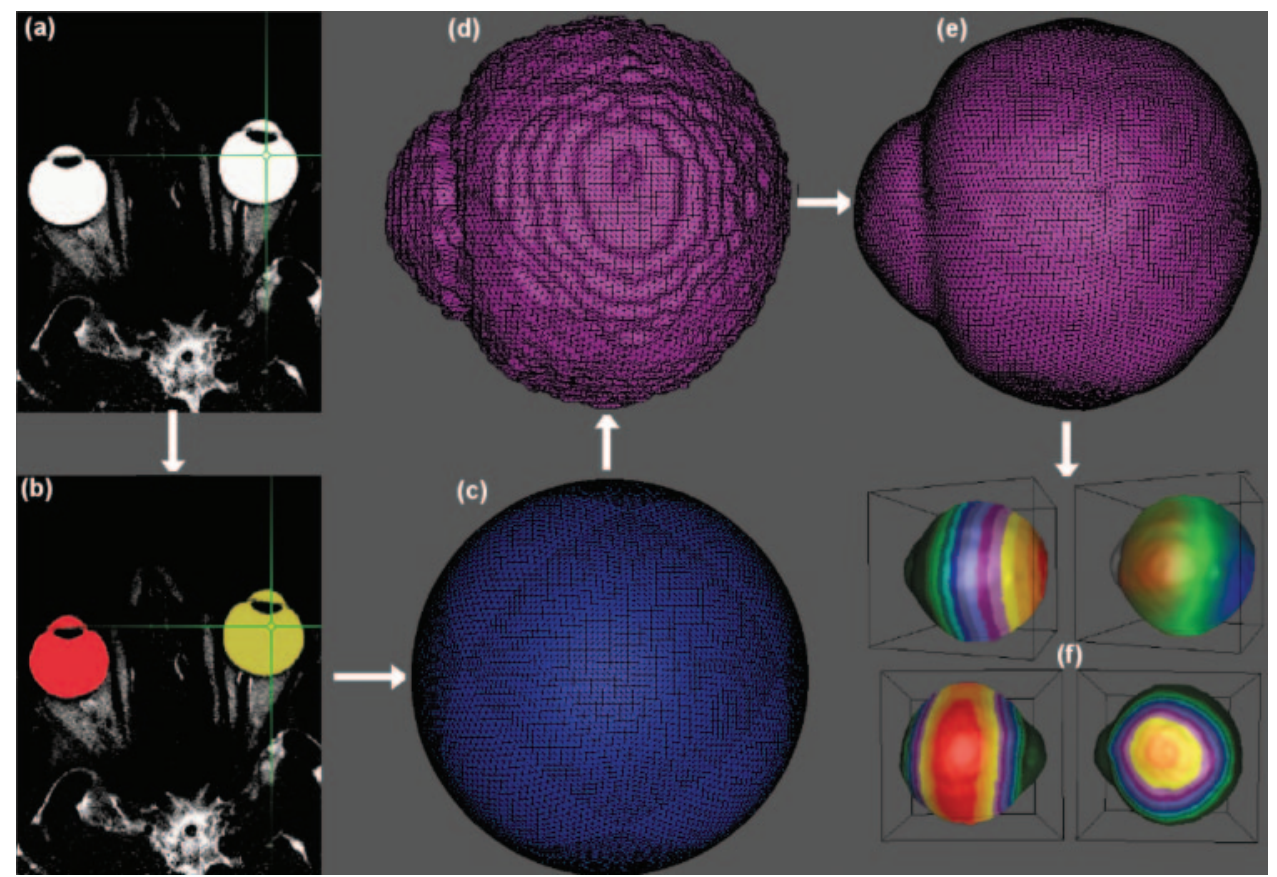


(3-Tesla Trio; Siemens, Erlangen, Germany). All participants gave informed consent consistent with the Declaration of Helsinki for research involving human subjects. The Ethics Committee of Aston University approved the study and written, informed consent was obtained from subjects after the nature of the study was explained to them. Note that to demonstrate the power of the technique, participants were chosen who had a wide variety of refractive errors, ranging from hyperopia through emmetropia to myopia. Six of the participants (subjects S1-S6; Table 1) were otherwise healthy, with no ocular disease. The seventh participant, $\mathrm{S} 7$, had Marfan syndrome, which is usually characterized by a high degree of myopia. MRIs were collected with an eight-channel phased-array head coil, which has the advantage of rapid scanning of both eyes simultaneously with a high signal-tonoise ratio. To provide a high-contrast delineation of the edges of the eye, a $\mathrm{T}_{2}$-weighted scan was performed with a half-acquisition turbo spin-echo sequence (HASTE) ${ }^{27,28}$ with the following parameters ${ }^{29,30}$ : 35 to 45 slices; $512 \times 512$ matrix; 256 -mm field of view; 1 -mm slice thickness; TR, $1240 \mathrm{~ms}$; TE, $124 \mathrm{~ms}$; flip angle, $150^{\circ}$; 6 averages; $4 / 8$ partial-phase acquisition; GRAPPA (genealized autocalibrating partially parallel acquisition) acceleration factor of 4 . Voxels within these images therefore have a $0.5 \times 0.5 \times 1-\mathrm{mm}$ intrinsic resolution. The sequence is designed to yield images in which the fluid-filled images of the eye are hyperintense compared with the rest of the head and, as a spin-echo sequence, susceptibility distortions and artifacts are minimized. In addition, local shimming of the region around the eyes was performed before each scan. This procedure is designed to correct for spatial variations in the magnetic field strength around the eyes, which would lead to geometric distortions. Previous studies of eye shape using spin-echo MR sequences have demonstrated negligible spatial distortions in the region around the eyes in a constructed model of the head. ${ }^{25}$ The scan time for each subject was only 5 minutes 40 seconds, during which they were asked to fixate on a distant spot viewed through a mirror mounted on the head coil.

\section{Segmentation Analysis}

MRIs were analyzed by using a specially modified version of the freeware software package mri3 dX (www.jiscmail.ac.uk/lists/mri3dx. html), developed by one of the authors (KDS). Figure 1 shows a schematic of the analysis pipeline. The images are loaded into mri3 dX (Fig. 1a) and it can be seen that the eyes are much brighter than any surrounding tissue. This means that the eyes can automatically be detected in the image, ${ }^{24}$ and voxels in each eye can be labeled with a 3-D flood-filling algorithm (Fig. 1b). In the figure, it can be seen that each eye was shaded with a different color (yellow, right eye; red, left eye), a method used to delineate multiple objects in the software. Both the anterior and posterior chambers of the eye were shaded. At this point, the operator manually identifies the anterior pole of each eye (referenced to the posterior corneal surface) and may also correct the shaded volumes, if necessary.

\section{Polygonal Mesh Construction}

A triangular mesh is then constructed that approximates the eye's shape (Figs. 1c-e). Many different algorithms exist for this purpose, but we chose to use a simplified version of a shrink-wrapping technique ${ }^{31}$ in which a model of a sphere is first constructed by using a mesh of 32,768 triangular polygons (Fig. 1c). The position and radius of this sphere is chosen to envelop the shaded eye voxels completely.

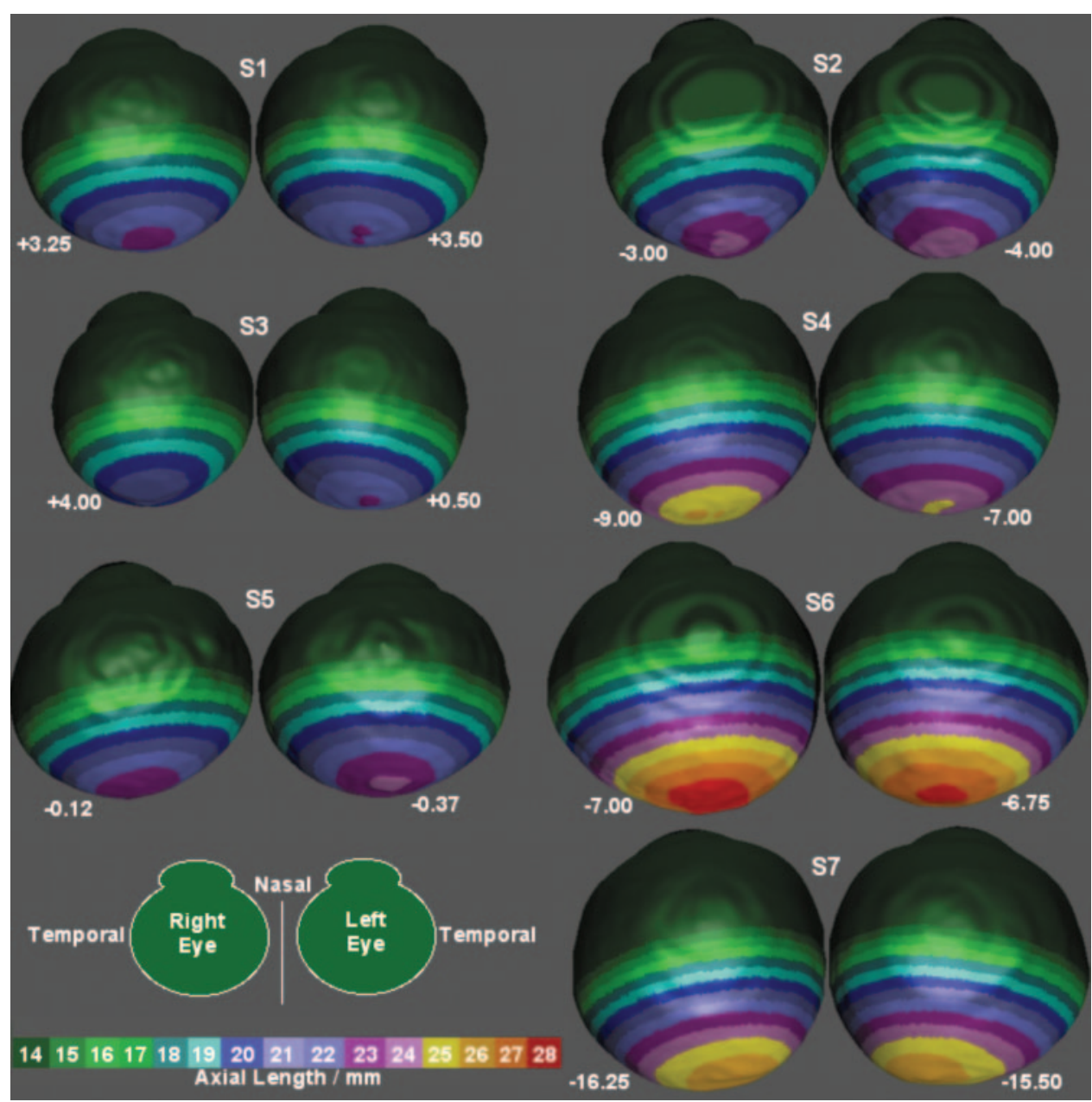

FIGURE 2. A depiction of the MRIdefined models of the 14 eyes studied of the seven participants (S1-S7). S7 had Marfan syndrome. The eye models are shaded with reference to axial distance from the corneal pole. The number near the posterior pole of each eye is the mean spherical distance refractive error for that eye. Bottom left: the orientation of each of the seven subfigures, together with a color-scale describing the mapping of axial length to each color. Note that each color actually represents a 1-mm range. The displayed numeral represents the lower boundary. Each eye is rotated slightly around the nasal-temporal axis to reveal the posterior pole of the eye. 
The vertices of each polygon are then shrunk toward the geometric center of the eye in an iterative fashion, until each vertex intersects a shaded voxel. The resultant polygonal model is wrapped to the shape of the eye (Fig. 1d) and has a corrugated appearance because of tight wrapping to each individual surface voxel. However, we can use the fact that we know the surface of the eye should be a relatively smooth structure. Hence, the polygonal mesh can be smoothed by using local averaging of the vertex positions to give a better approximation of the shape of the eye (Fig. 1e).

\section{Visualization}

Once the surface of the eye has been constructed, various morphometric parameters can be calculated and superimposed on the model using a color-coded palette (Fig. 1f). A variety of parameters can be depicted, including the total 3-D Pythagorean distance from the posterior corneal pole, the distance from the corneal pole along the longitudinal axis of the eye, the deviation from the mean sphere and horizontal-vertical distances from the longitudinal axis of the eye. These shaded 3-D models were visualized in the freeware package, Geomview (www.geomview.org), which allows the model to be rotated and viewed in any position and orientation and greatly aids in the understanding of each eye shape.

\section{Standardized Coordinate System}

For many of these displayed parameters, it is important to define a standardized 3-D coordinate system, defined herein by the unit vectors $\mathbf{u}, \mathbf{v}$, and $\mathbf{w}$, for each eye. The first axis we defined, $\mathbf{v}$, is the longitudinal symmetry axis of the eye, which is equivalent to the visual or optical axis. This is calculated as a line joining the geometrical center of the eye with the anterior corneal pole. We have found that projecting this line back to the rear of the eye gives a good approximation of where we expect the fovea to be located. Defining standardized horizontal (nasal-temporal) and vertical (inferior-superior) axes for all eyes is more problematic, as each eye is approximately spherically symmetric about v. However, we have found the following approach to be robust: First, an estimate of the horizontal axis, $\mathbf{e}$, is made as a line joining the geometric centers of the two eyes. Because of the slight horizontal divergence of the eyes, $\mathbf{e}$ is not guaranteed to be perpendicular to $\mathbf{v}$, so a refinement is needed. First, the vertical axis, $\mathbf{w}$, is calculated using the formula $\mathbf{w}=\mathbf{e} \times \mathbf{v}$. Then, the final horizontal axis, $\mathbf{u}$, is calculated using $\mathbf{u}=\mathbf{v} \times \mathbf{w}$.

The main advantage of using the shrink-wrapping approach just described is that each triangular polygon on the eye surface directly maps back to a position on a sphere in the standardized coordinates described. This means that, in principle, group statistical analysis of eye shape could be performed in a standard sphere template space, in much the same way as in the analysis of group functional brain images. ${ }^{32}$ This ability to perform group analysis will be important in large studies, such as those determining outcome measures in clinical trials.

\section{Validation}

One concern with any structural MRI study, especially when we are approaching the limit of the device's resolution, is whether the images are subject to significant spatial distortion. As described earlier, we have tried to minimize this risk by using spin-echo sequences and local shimming, but independent validation is essential. Although some of this can be done by quality-assurance testing using phantoms, which is done routinely in our laboratory, this does not guarantee that the images are spatially accurate in vivo. However, there are several tools for noninvasive ocular biometry that can provide distance measures for validating the accuracy of our 3-D models. For example, partial coherence interferometry (PCI) can measure the axial length of the eye to better than $0.02 \mathrm{~mm} .{ }^{33}$ The axial length of all 14 eyes was therefore also measured with a PCI-based device (IOLMaster; Carl Zeiss Meditec, Inc., Jena, Germany), and these measurements were compared with those determined from the MRI models described earlier.

\section{Results}

Figure 2 shows the 14 MRI-derived eye models for all seven participants in this study, whereas Table 1 provides quantitative biometric data for each eye. Each model is color coded with axial distance from the posterior corneal pole. Several notable features are evident. First, there is substantial variation in the absolute size of the eyes. Compare, for example the left eyes of $\mathrm{S3}$ and $\mathrm{S5}$, both of which are approximately emmetropic but are very different in terms of size and shape. There are also variations in the sphericity of the eyes, with some appearing approximately spherical (S1), others clearly oblate (S5), and one slightly prolate (S2). There are also nasaltemporal asymmetries evident in some subjects, such as $\mathbf{S 6}$.

It is also evident from Figure 2 and Table 1 that, in the small sample of participants in this study, there is no clear dependency of refractive error on axial length. The models are consistent therefore with the significant intersubject variation previously reported in relation to axial length and its correlation with refractive error. For example, the left eye of S4 and the right eye of $\mathrm{S} 6$ have the same refractive error $(-7.00 \mathrm{D})$, but are clearly different in axial length and symmetry of eye shape. The former is made more evident in Figure 3, where these two eye models are directly compared. For the right eyes of S4 and S6, the depth of myopia is less than expected from the total axial length, suggesting that axial myopia is offset by increased refractive power of the cornea and/or lens. In contrast, for three subjects with right eye axial lengths (as measured by PCI) that are approximately the same (i.e., S1, S2, and S5) there is very substantial variation in refractive error (respectively, +3.25 $\mathrm{D} ;-3.00 \mathrm{D} ;-0.12 \mathrm{D})$ and overall eye shape and size.

The utility of 3-D representation is further demonstrated for S7, the participant with Marfan syndrome, who had extremely

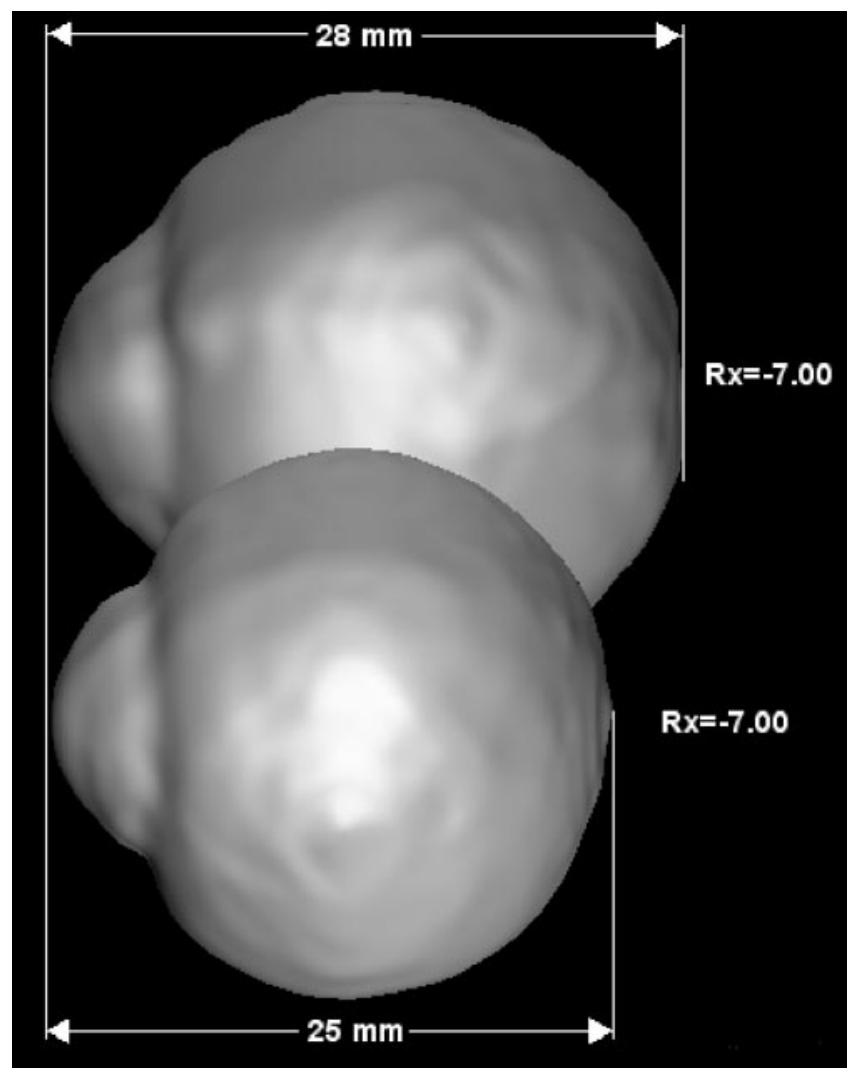

FigURE 3. A comparison of the MRI models for two eyes with the same mean sphere refractive error of $-7.00 \mathrm{D}$. Top: model of the right eye of S6; bottom: model of the left eye of S4. 

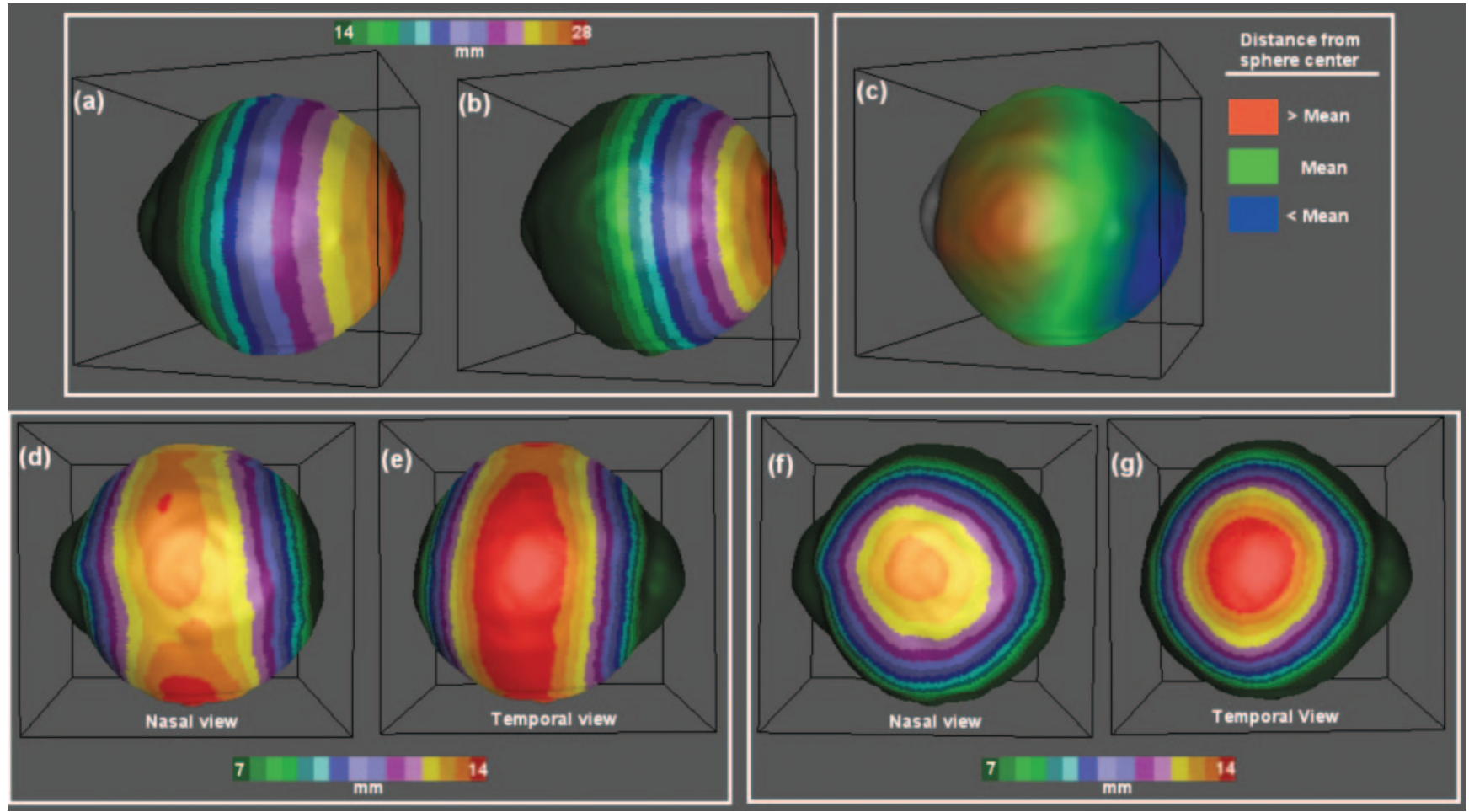

FiguRE 4. A depiction of multiple morphometric parameters for the right eye of S6 ( $-7.00 \mathrm{D})$. (a) The total (Pythagorean) 3-D distance from the corneal pole; (b) axial distance from the same point. (c) Deviation from sphericity, with colors representing differences from the mean radius of the best-fit sphere to the posterior portion of the eye model, not including the anterior chamber. (d-g) The distance from the longitudinal axis of the eye, with (d) and (e) showing total distance from the axis, and (f) and (g) showing only the horizontal distance component (i.e., the distance along the nasal-temporal axis). The bottom panels demonstrate a clear asymmetry in this eye, with the eye more bulbous in the temporal direction.

high myopia. Despite this high myopia, S7 did not have particularly long eyes; however, examination of the lenses of $\mathrm{S} 7$ in the original MRI images indicated spherophakia, which is associated with this condition. The small size of the lens, compared with our measurement voxel size, is such that accurate quantification of the lens dimensions is not possible using the coil and sequence used in this study.

Figure 4 demonstrates the variety of parameters that can be displayed on the each eye model, for the right eye of S6. The eye is clearly oblate (Fig. 4c) and in addition shows a differen- tial bulge in the temporal region (Figs. $4 \mathrm{f}, 4 \mathrm{~g}$ ). This is further quantified in Figure 5 , in which it can be seen that in the equatorial region of the eye $(\sim 15 \mathrm{~cm}$ from the anterior pole along the axis of the eye), the nasal segments of the eye (green points) are slightly closer to the longitudinal axis of the eye than the temporal points (blue/purple points). Nasal-temporal asymmetries in the shape of eyes of white individuals have been demonstrated by computer modeling. ${ }^{34}$

In Figure 6, a validation is made of the spatial accuracy of the reconstructed models. This graph shows the relationship

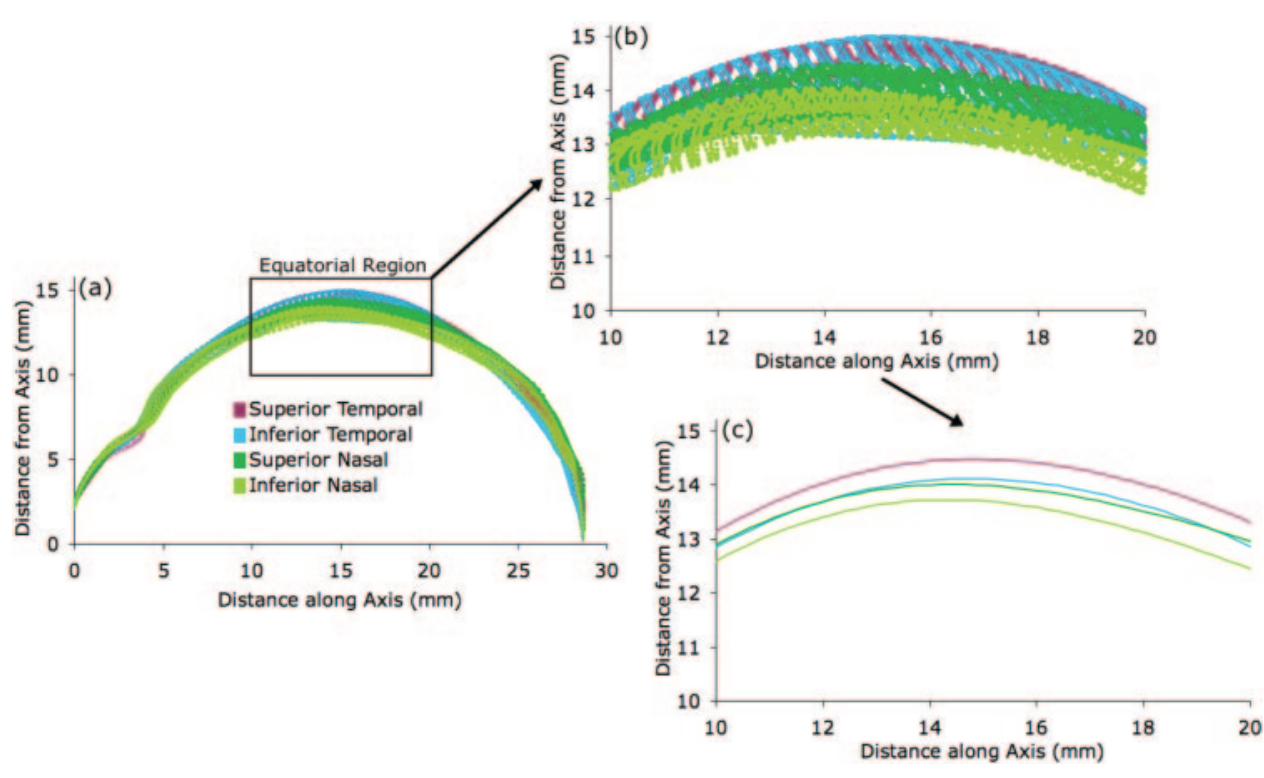

Figure 5. The profile of the right eye of S6. Each point represents one vertex of each of the polygons in the mesh model of the eye. The points are color-coded depending on which quadrant of the surface includes the vertex. The $x$-axis represents the distance along the longitudinal axis of the eye, from the corneal pole on the left to the fovea on the right. The $y$-axis represents each vertex's distance from the longitudinal axis of the eye. (a) All points in the mesh model of the whole eye. (b) A magnified version of the equatorial region of the eye. (c) Curve-fits (sixthorder polynomial) for the mesh points in each quadrant of the eye, shown for the magnified region. 

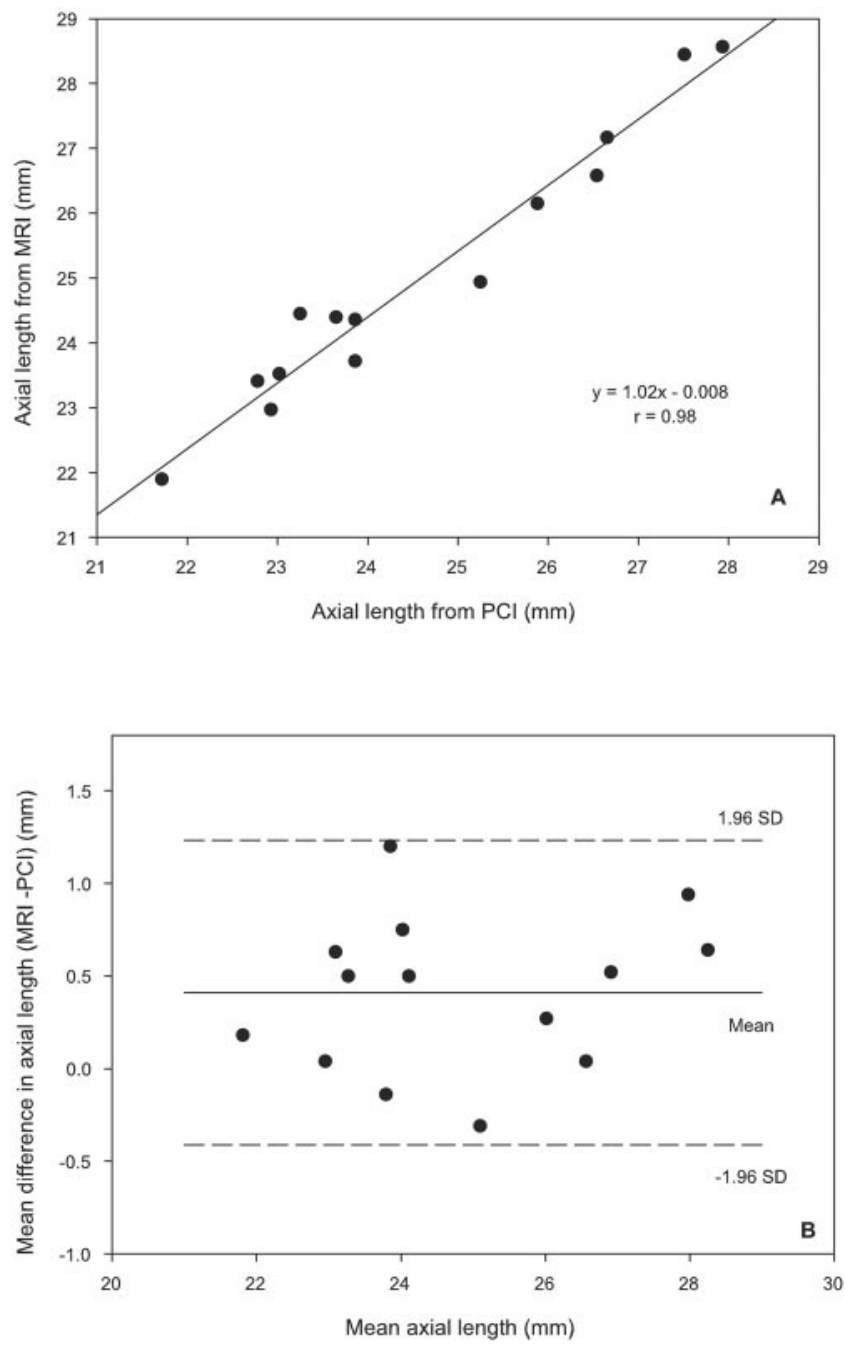

FiguRE 6. (A) The relationship between the axial length of the eye, as measured using PCI ( $x$-axis), and the axial length, as determined by the MRI method ( $y$-axis). (B) A plot of the difference between the axial lengths as determined by the MRI method and using PCI ( $y$-axis) versus the mean of the two techniques (x-axis).

between axial length, as derived by MRI, and axial length measured using PCI. ${ }^{33}$ In the 14 eyes measured in this study, there was good concordance between the two methodologies.
However, a clear bias was evident between the two measures, with the MRI determined lengths being longer than those determined by PCI (mean difference, $0.41 \mathrm{~mm}$ ). There are various possibilities for the source of this systematic bias, with the most likely explanation related to the thickness $(0.5 \times$ $0.5 \times 1.0 \mathrm{~mm}$ ) of the voxels acquired in the MRI acquisition. The polygonal mesh used to represent the eye wraps tightly to the outside of these voxels, so any lengths determined by the mesh will tend to overestimate the true distance. Another possible explanation relates to the possibility that the length of the eye changes slightly as the head changes orientation between the upright (PCI) and supine (MRI) positions. In addition, it should be noted that there are some potential inaccuracies inherent in the use of $\mathrm{PCI}$ in measurement of axial length of the eye. Most important, PCI requires an accurate estimation of the refractive indices of each ocular component to convert optical path length to the required geometrical length. One advantage of MRI-based techniques is that no such estimation is required.

Finally, the repeatability of the technique was assessed by performing 10 different MR measurement sessions on the same participant, who was completely removed from the scanner between sessions. Length, width, and volume measures of both eyes were then calculated from the constructed 3-D mesh models for each of the 10 sessions (Table 2). The technique shows reasonable intersession robustness, with a repeatability that is consistent with the size of the voxels in the original scans.

\section{Discussion}

Our study demonstrates a novel approach to forming 3-D models of the complete eye from ocular MRIs. The technique complements the measurements of ocular dimensions provided by techniques such as ultrasound, PCI ${ }^{33}$ and optical low-coherence reflectometry ${ }^{35}$ for longitudinal measurements of axial length and the reconstruction of 2-D representations of posterior retinal shape by the addition of off-axis refraction ${ }^{36}$ and computer modeling. ${ }^{34}$ MRI has been used for many aspects of ocular physiology $y^{37-39}$; however, in terms of measurement of ocular dimensions, it has been predominantly restricted to measurements of the accommodative apparatus of the anterior segment, ${ }^{40,41}$ the dimensions of the extraocular muscles ${ }^{42}$ and limited 2-D/3-D reconstructions of the posterior segment in myopia. ${ }^{22,23,25,26}$ Recent studies using large subject samples have demonstrated that, in general, myopic stretch is characteristically relatively prolate. However, as in the data presented herein, substantial intersubject variation was evident (Miller et

TABLE 2. Ten MRI Scans and Analyses on Eyes of One Subject

\begin{tabular}{|c|c|c|c|c|c|c|}
\hline Repeat & $\begin{array}{c}\text { RE Axial Length } \\
\text { (mm) }\end{array}$ & $\begin{array}{c}\text { RE Width (Radius) } \\
\text { (mm) }\end{array}$ & $\begin{array}{l}\text { RE Volume } \\
\left(\mathrm{mm}^{3}\right)\end{array}$ & $\begin{array}{c}\text { LE Axial Length } \\
(\mathrm{mm})\end{array}$ & $\begin{array}{c}\text { LE Width (Radius) } \\
\text { (mm) }\end{array}$ & $\begin{array}{l}\text { LE Volume } \\
\left(\mathrm{mm}^{3}\right)\end{array}$ \\
\hline 1 & 24.06 & 13.31 & 6531.00 & 24.61 & 13.85 & 6734.00 \\
\hline 2 & 23.79 & 13.25 & 6413.00 & 24.89 & 13.77 & 6664.00 \\
\hline 3 & 23.08 & 13.27 & 6416.00 & 24.73 & 13.82 & 6726.00 \\
\hline 4 & 23.73 & 13.63 & 6741.00 & 24.66 & 13.64 & 6786.00 \\
\hline 5 & 23.88 & 13.44 & 6460.00 & 24.70 & 13.90 & 6666.00 \\
\hline 6 & 23.89 & 13.29 & 6471.00 & 24.66 & 13.87 & 6764.00 \\
\hline 7 & 23.83 & 13.32 & 6489.00 & 23.73 & 13.89 & 6770.00 \\
\hline 8 & 23.99 & 13.15 & 6350.00 & 23.79 & 13.84 & 6671.00 \\
\hline 9 & 23.79 & 13.57 & 6474.00 & 23.71 & 13.73 & 6774.00 \\
\hline 10 & 23.73 & 13.21 & 6297.00 & 23.63 & 13.80 & 6626.00 \\
\hline Mean & 23.78 & 13.34 & 6464.20 & 24.31 & 13.81 & 6718.10 \\
\hline SD & 0.27 & 0.16 & 119.16 & 0.52 & 0.08 & 56.95 \\
\hline SE & 0.08 & 0.05 & 37.68 & 0.16 & 0.03 & 18.01 \\
\hline
\end{tabular}

RE, right eye; LE, left eye. 
al. IOVS 2004;45:ARVO E-Abstract 2388), with the horizontal meridians appearing to stretch less than the vertical meridians. ${ }^{25}$

The use of full 3-D ocular models from MRI data will provide valuable biometric information for current and future initiatives in myopia research and will help determine the clinical significance of human corollaries of myopic growth in animal eyes, ${ }^{21}$ which have formed the biological basis for recent clinical trials on myopia control in children. ${ }^{43,44}$ The ability to stratify and analyze eye shape, volume, and sphericity will further extend our understanding of which specific biometric parameters predispose development of myopia in emmetropic children, especially in those exposed to environmental and/or hereditary factors.

The methodologies described herein can be extended to provide further information about the eye. For example, $\mathrm{T}_{1^{-}}$ weighted MRIs showing structures such as the choroid and sclera could be coregistered with the 3-D models to show spatial variation in etiological parameters, such as choroidal and scleral thickness. ${ }^{45}$ In addition, the spatial resolution of these 3-D models may be further enhanced by the use of optimized surface coils in the MR acquisition, rather than the head coil used in this study. This may provide the resolution needed to measure choroidal and scleral thicknesses. In addition, recent studies have demonstrated the utility of highresolution MRI in characterizing the shape of elements of the anterior eye, such as the crystalline lens. ${ }^{40,41}$ In principle, the meshing algorithms described herein could be used to parametrically model the shape of anterior components such as the lens. However, the small size of these structures again makes it necessary to use optimized high-resolution surface coils.

Measurement of ocular volume is particularly relevant to our understanding of choroidal function in myopia and its relationship to choroidal blood flow. ${ }^{46}$ A composite 3-D representation of the posterior segment will allow us to address better the intriguing questions concerning asymmetry of ocular stretching ${ }^{34}$ and the effect of peripheral and central stretching on ocular aberrations, receptor orientation, and image quality. ${ }^{47-49}$ Finally, by providing a means of correlating specific characteristics of retinal disease with ocular stretching, the technique could aid the clinical assessment of ocular risk in high myopia.

\section{References}

1. Curtin BJ. The Myopias: Basic Science and Clinical Management. Philadelphia: Harper \& Row; 1985.

2. Morgan I, Rose K. How genetic is school myopia? Prog Retin Eye Res. 2005;24:1-38.

3. Negrel AD, Maul E, Pokharel GP, Zhao JL, Ellwein LB. Refractive error study in children: sampling and measurement methods for a multicountry survey. Am J Opbthalmol. 2000;129:421-426.

4. Dandona R, Dandona L, Srinivas M, et al. Refractive error in children in a rural population in India. Invest Opbthalmol Vis Sci. 2002;43:615-622.

5. Murthy GVS, Gupta SK, Ellwein LB, et al. Refractive error in children in an urban population in New Delhi. Invest Opbthalmol Vis Sci. 2002;43:623-631.

6. Naidoo KS, Raghunandan A, Mashige KP, et al. Refractive error and visual impairment in African children in South Africa. Invest Ophthalmol Vis Sci. 2003;44: 3764-3770.

7. He M, Zeng J, Liu Y, Xu J, Pokharel GP, Ellwein LB. Refractive error and visual impairment in urban children in southern China. Invest Opbthalmol Vis Sci. 2004;45:793-799.

8. Wallman J. Vision: nature and nurture of myopia. Nature. 1994; 371:201-202.

9. Zadnik K, Manny RE, Yu JA, et al. Ocular component data in schoolchildren as a function of age and gender. Optom Vis Sci. 2003;80:226-236.
10. Jones LA, Mitchell GL, Mutti DO, Hayes JR, Moeschberger ML, Zadnik K. Comparison of ocular component growth curves among refractive error groups in children. Invest Opbthalmol Vis Sci. 2005;46:2317-2327.

11. Wallman J, Winawer J. Homeostasis of eye growth and the question of myopia. Neuron. 2004;43:447-468.

12. Gilmartin B. Myopia: precedents for research in the twenty-first century. Clin Exp Opbthalmol. 2004;32:305-324.

13. Vongphanit J, Mitchell P, Wang JJ. Prevalence and progression of myopic retinopathy in an older population. Ophthalmology. 2002; 109:704-711.

14. Wiesel TN, Raviola E. Myopia and eye enlargement after neonatal lid fusion in monkeys. Nature (Lond). 1977;266:66-68.

15. Raviola E, Wiesel TN. An animal model of myopia $N$ Engl J Med. 1985;312:1609-1615.

16. Wallman J, Gottlieb MD, Rajaram V, Fugate-Wentzek LA. Local retinal regions control local growth and myopia. Science. 1987; 237:73-77.

17. Stone RA, Lin T, Laties AM, Iuvone PM. Retinal dopamine and form-deprivation myopia. Proc Natl Acad Sci USA. 1989;86:704706.

18. Stone RA, Lin T, Laties AM. Muscarinic antagonist effects on experimental chick myopia Exp. Eye Res. 1991;52:755-758.

19. Norton TT. Animal models of myopia: learning how vision controls the size of the eye. Inst Lab Anim Res J. 1999;40:59-77.

20. Hung LF, Crawford ML, Smith EL. Spectacle lenses alter eye growth and the refractive status of young monkeys. Nat Med. 1995;1:761765 .

21. Stone RA, Flitcroft DI. Ocular shape and myopia. Ann Acad Med Singapore. 2004;33:7-15.

22. Chen JF, Elsner AE, Burns SA, et al. The effect of eye shape on retinal responses. Clin Vision Sci. 1992;7:521-530.

23. Cheng H-M, Singh OS, Kwong K, Xiong J, Woods BT, Brady TJ. Shape of the myopic eye as seen with high-resolution magnetic resonance imaging. Optom Vis Sci. 1992;69:698-701.

24. Chau C, Fung K, Pak K, Yap M. Is eye size related to orbit size in human subjects? Opbthalmic Pbysiol Opt. 2004;24:35-40.

25. Atchison DA, Jones CE, Schmid KL. Eye shape in emmetropia and myopia Invest Ophthalmol Vis Sci. 2004;45:3380-3386.

26. Atchison DA, Pritchard N, Schmid KL, Scott DH, Jones CE, Pope JM. Shape of the retinal surface in emmetropia and myopia. Invest Opbthalmol Vis Sci. 2005;46:2698-2707.

27. Patel MR, Klufas RA, Alberico RA, Edelman RR. Half-fourier acquisition single-shot turbo spin-echo (HASTE) MR: comparison with fast spin-echo MR in diseases of the brain. Am J Neuroradiol. 1997; $18: 1635-1640$

28. Penzkofer AK, Pfluger T, Pochmann Y, Meissner O, Leinsinger G. MR imaging of the brain in pediatric patients: diagnostic value of HASTE sequences. Am J Roentgenol. 2002;179:509-514.

29. McRobbie DW, Moore EA, Graves MJ, Prince MR. MRI from Picture to Proton. Cambridge, UK: Cambridge University Press; 2003.

30. Hornak JP. The Basics of MRI: a Hypertext Book on Magnetic Resonance Imaging. 1996, 1997. Rochester, NY: Rochester Institute of Technology. Available online at: http://www.cis.rit.edu/ htbooks/mri

31. van Overveld K, Wyvill B. Shrinkwrap: An efficient adaptive algorithm for triangulating an iso-surface. Visual Comput. 2004;20: 362-379.

32. Fox PT. Spatial normalization origins: objectives, applications, and alternatives. Hum Brain Mapp. 1995;3:161-164.

33. Santodomingo-Rubido J, Mallen EAH, Gilmartin B, Wolffsohn JS. A new non-contact optical device for ocular biometry. Br J Ophthalmol. 2002;86:458-462.

34. Logan NS, Gilmartin B, Wildsoet CF, Dunne MCM. Posterior retinal contour in adult human anisomyopia. Invest Ophthalmol Vis Sci. 2004; $45: 2152-2162$

35. Schmid GF. Variability of retinal steepness at the posterior pole in children 7-15 years of age. Curr Eye Res. 2003;27:61-68.

36. Seidemann A, Schaeffel F, Guirao A, Lopez-Gil N, Artal P. Peripheral refractive errors in myopic, emmetropic, and hyperopic young subjects. J Opt Soc Am A. 2002;19:2363-2373. 
37. Berkowitz BA, Roberts R, Luan HM, Peysakhov J, Mao XZ, Thomas KA. Dynamic contrast-enhanced MRI measurements of passive permeability through the blood retinal barrier in diabetic rats. Invest Ophthalmol Vis Sci. 2004;45:2391-2398.

38. Trick GL, Berkowitz BA. Retinal oxygenation response and retinopathy. Prog Retin Eye Res. 2005;24:259-274.

39. Demer JL, Clark RA, Engle EC. Magnetic resonance imaging evidence for widespread orbital dysinnervation in congenital fibrosis of extraocular muscles due to mutations in KIF21A. Invest $O p b$ thalmol Vis Sci. 2005;46:530-539.

40. Strenk SA, Semmlow JL, Strenk LM, Munoz P, Gronlund-Jacob J, DeMarco JK. Age-related changes in human ciliary muscle and lens: a magnetic resonance image study. Invest Ophthalmol Vis Sci. 1999; 40:1162-1169.

41. Strenk SA Strenk LM, Semmlow JL, DeMarco JK. Magnetic resonance imaging study of the effects of age and accommodation on the human lens cross-sectional area. Invest Ophthalmol Vis Sci. 2004;45:539-545.

42. Miller JM. Functional anatomy of normal human rectus muscles. Vision Res. 1989;29:223-240.

43. Gwiazda JE, Hyman L, Norton TT, et al. Accommodation and related risk factors associated with myopia progression and their interaction with treatment in COMET children. Invest Ophthalmol Vis Sci. 2004;45:2143-2151.

44. Siatkowski RM, Cotter S, Miller JM, Scher CA, Crockett RS, Novack GD. Safety and efficacy of $2 \%$ pirenzepine ophthalmic gel in children with myopia: a 1-year, multicenter, double-masked, placebo controlled parallel study. Arch Ophthalmol. 2004;122:16671674 .

45. Wallman J, Wildsoet CF, Xu AM, et al. Moving the retina: choroidal modulation of refractive state. Vision Res. 1995;35:37-50.

46. Fitzgerald MEC, Wildsoet CF, Reiner A. Temporal relationship of choroidal blood flow and thickness changes during recovery from form deprivation myopia in chicks. Exp Eye Res. 2002;74:561570 .

47. He JC, Sun P, Held R, Sun X, Gwiazda JE. Wavefront aberrations in eyes of emmetropic and moderately myopic school children and young adults. Vision Res. 2002;42:1063-1070.

48. Chui TYP, Yap MKH, Chan HHL, Thibos LN. Retinal stretching limits peripheral acuity in myopia. Vision Res. 2005;45:593-605.

49. Vera-Diaz FA, McGraw PV, Strang NC, Whitaker D. A psychophysical investigation of ocular expansion in human eyes. Invest $O p b$ thalmol Vis Sci. 2005;46:758-763.

\section{$\begin{array}{llllll}\mathbf{E} & \mathbf{R} & \mathbf{R} & \mathbf{A} & \mathbf{T} & \mathbf{A}\end{array}$}

Erratum in: "Preservation and Expansion of the Primate Keratocyte Phenotype by Downregulating TGF- $\beta$ Signaling in a Low-Calcium, Serum-Free Medium" by Kawakita et al. (Invest Opbthalmol Vis Sci. 2006;47:1918-1927).

Due to a printer error, the following corrections were not made to the article prior to publication.

The Disclosure for R. Smiddy is TissueTech, Inc. (C) and for J.-M. Parel and C.-Y. Liu is None.

In the second sentence of the Discussion, References 5-7 should be cited after CD34. The sentence should read, "Subsequently, other markers for keratocytes have been reported, including keratan sulfate-containing proteoglycans, ${ }^{3,4,34}$ such as keratocan, ${ }^{4,15,35} \mathrm{CD} 34,{ }^{5-7}$ and ALDH. ${ }^{4,36 ", ~}$

Erratum in: "Effects of Low AIPL1 Expression on Phototransduction in Rods" by Makino et al. (Invest Ophthalmol Vis Sci. 2006;47:2185-2194).

Due to a printer error, certain units in the legends of Figure 3 and Table 2 were printed fL/s instead of fL-s.

The first sentence in (A) of the Figure 3 legend should read, "A saturating, 28-fL-s flash of white light, producing an estimated 11,700 photoisomerizations per rod, was given at time 0. ."

The first sentence in $(\mathbf{C})$ of the Figure 3 legend should read, "Responses to paired saturating flashes of 90 fL-s delivered 4 minutes apart in 12- to 20-week-old mice."

The last sentence of the Table 2 legend should read, "Implicit times were measured in different mice at 8 to 20 weeks with a subsaturating flash of $0.2 \mathrm{fL}$-s that produced an estimated 80 photoisomerizations per rod."

In Table 2, the units for "a-Wave implicit time" are milliseconds and should read "(ms)." 